\title{
Comparison of cytokine profiles in bronchoalveolar lavage fluid of mice exposed to respiratory and contact sensitizers
}

\author{
Takashi Mori, Yoshiharu Tanimoto, Mika Ota, Takaomi Masakado, Sachiko Kitamoto, \\ Koichi Saito, Naohiko Isobe and Hideo Kaneko \\ Environmental Health Science Laboratory, Sumitomo Chemical Co., Ltd., 3-1-98, Kasugadenaka, Konohana-ku, \\ Osaka 554-8558, Japan
}

(Received October 20, 2011; Accepted December 30, 2011)

\begin{abstract}
Respiratory sensitization to certain low molecular weight chemicals is a big concern for workers, but unfortunately there are no validated animal models to allow identification of sensitizing chemicals in the environment. In the present study, dermally sensitized and intratracheally challenged mice were used to investigate effective indicators of respiratory sensitizers. Changes in levels of total serum IgE and nine cytokines (G-CSF, IL-4, IL-5, IL-6, IL-12(p70), IL-13, IFN- $\gamma$, MCP-1 and TNF- $\alpha$ ) in bronchoalveolar lavage fluid (BALF) were analyzed in BALB/c mice exposed to respiratory sensitizers (phthalic anhydride (PA); diphenylmethane-4,4'-diisocyanate (MDI); toluene diisocyanate (TDI); chloramine- $\mathrm{T}(\mathrm{CH})$; and piperazine $(\mathrm{PI})$ ) or contact sensitizers (2,4-dinitrochlorobenzene (DNCB); and oxazolone (OXA)). Non-sensitized mice were treated dermally with solvents and challenged intratracheally with the respective test chemicals as solvent controls. Increases in total serum IgE levels were observed in all treated mice, with apparent differences in cytokine profiles. PA caused statistically significant increases in Th2 cytokines, IL-4, IL-5 and IL-13, compared with the control. IL-5 was also found to be increased with $\mathrm{CH}$. The other three respiratory sensitizers caused statistically significant increases in IL-13. In contrast, no change was apparent with contact sensitizers, DNCB and OXA, in these Th2 cytokines. Increases in the Th2 cytokines indicate that all five respiratory sensitizers induced immune responses in lungs. Interestingly, elevation of G-CSF levels in BALF appeared with all five respiratory sensitizers but not the two contact sensitizers. The findings suggest that G-CSF could be effective to identify respiratory sensitizers in animal models.
\end{abstract}

Key words: Cytokines, Bronchoalveolar lavage fluid, Respiratory sensitizer, Contact sensitizer, Dermal sensitization, Intratracheal challenge

\section{INTRODUCTION}

Repeated exposure to certain low molecular weight (LMW) chemicals causes allergic responses in the human airway. These chemicals include diisocyanates and acid anhydrides that are generally used to make resins and polymers, which is therefore a major concern for workers in many industries (Tarlo, 2003). The immune response features IgE production, cell infiltration and subsequent inflammation in airways, leading to respiratory troubles such as asthma (Mapp et al., 2005). Epidemiological studies have shown that respiratory sensitizers induce complex symptoms, for example, diphenylmethane-4,4'diisocyanate (MDI), a well-known example, which caus- es allergic asthma with pathophysiological features such as eosinophilia and reactive airway dysfunction syndrome in the lungs (Leroyer et al., 1998; Hur et al., 2008). In addition, respiratory sensitizers may exhibit IgE-dependent or-independent responses (Kimber et al., 1998) and mechanisms of LMW chemical-induced respiratory allergy remain to be defined.

At present, there are no validated methods to experimentally identify respiratory sensitizers and their classification is mainly based on epidemiological studies of human populations (Kimber et al., 2007). However, various animal models have been proposed (Arts and Kuper, 2007; Boverhof et al., 2008). Previous studies showed the effectiveness of dermal application as a route of sensiti-

Correspondence: Takashi Mori (E-mail: morit14@sc.sumitomo-chem.co.jp) 
zation and cytokines as indicators (Dearman et al., 2000; Van et al., 2002). Respiratory allergy is generally considered to be based on Th2 immune responses and dermal application of several respiratory sensitizers was found to induce such responses in mice (Dearman and Kimber, 2001). In order to measure cytokines, draining lymph node cells and bronchoalveolar lavage fluid (BALF) may be used (Dearman et al., 2000; Van et al., 2002; Fukuyama et al., 2008). For example, lymph node cells have been collected from chemical sensitized mice and cultured with or without concanavalin A, a stimulation factor of the production of IL-4, and shown by Dearman and co-workers to respond to several respiratory sensitizers by secreting Th2 cytokines (IL-4, IL-5 and IL-13) (Dearman et al., 2000; Dearman and Kimber, 2001). Similarly, Th2 cytokines (IL-4, IL-5 and IL-13) are increased in BALF (Ban et al., 2006). Therefore, Th2 cytokines are considered to be effective for identification of respiratory sensitizing potentials of chemicals. However, few systematic studies on various cytokine responses caused by chemical respiratory sensitizers in lungs were reported. In addition, most studies used a limited battery of test chemicals such as diisocyanates and acid anhydrides for analysis of BALF cytokines.

The objectives of this study were to identify effective cytokines as markers for detecting respiratory sensitizing potentials of chemicals. In the study, we analyzed nine BALF cytokines (G-CSF, IL-4, IL-5, IL-6, IL-12(p70), IL-13, IFN- $\gamma$, MCP-1 and TNF- $\alpha$ ) and serum total IgE of dermally sensitized and intratracheally challenged mice using five known respiratory sensitizers and two contact sensitizers systematically. Our results provide useful information on the identification of respiratory sensitizers using BALF cytokines in animal models.

\section{MATERIALS AND METHODS}

\section{Animals}

Female BALB/c mice (6-7 weeks old) were purchased from Charles River (Kanagawa, Japan) and individually identified by marking with ink. They were housed in plastic cages containing wood-chips, and allowed food and water ad libitum. Before treatment, the mice were quarantined for 1 week and acclimatized in the room maintained on a 12-hr light-dark cycle. All of the animal experiments were conducted in accordance with current Japanese laws and the Guidelines for Proper Conduct of Animal Experiments (Science Council of Japan) and were approved by the Environmental Health Science laboratory IACUC of Sumitomo Chemical Co., Ltd.

\section{Chemicals}

Toluene diisocyanate (TDI) and acetone were purchased from Kanto Chemical (Tokyo, Japan); phthalic anhydride (PA), diphenylmethane-4,4'-diisocyanate (MDI), chloramine-T (CH), piperazine (PI), 2,4-dinitrochlorobenzene (DNCB), olive oil and diethyl ether from Nakalai Tesque (Kyoto, Japan); dimethyl sulfoxide (DMSO) from Dojindo Laboratories (Kumamoto, Japan); oxazolone (OXA) from Sigma-Aldrich (Tokyo, Japan); midazolam from Astellas Pharma (Tokyo, Japan); and medetomidine and butorphanol from Meiji Seika Kaisha (Tokyo, Japan). PA, MDI, TDI, DNCB and OXA were dissolved in 4:1 acetone:olive oil (AOO) for dermal application. PA was heated to aid solution in AOO using a water bath before application. $\mathrm{CH}$ and PI were dissolved in DMSO for dermal application. For intratracheal instillation, PA, CH, PI and OXA were dissolved in DMSO and mixed with olive oil, while MDI, TDI and DNCB were mixed with olive oil.

\section{Dermal application and intratracheal instillation}

For dermal application, 25\% (w/v) PA, 2\% (w/v) MDI, $1 \%(\mathrm{w} / \mathrm{v})$ TDI, $10 \%(\mathrm{w} / \mathrm{v}) \mathrm{CH}, 5 \%(\mathrm{w} / \mathrm{v}) \mathrm{PI}, 1 \%(\mathrm{w} / \mathrm{v})$ DNCB, $1 \%(w / v)$ OXA or vehicle (AOO or DMSO) were used (Table 1). Fifty $\mu$ l of chemical was applied on each shaved flank on days $0,1,6$ and 7 . On days 12, 13 and 14, $25 \mu 1$ aliquots were applied on the dorsum of both ears. Non-sensitized mice were similarly treated dermally with solvents. On day 27, $50 \mu \mathrm{l}$ of $0.05 \%$ (w/v) PA, $0.0001 \%$ (w/v) MDI, 0.001\% (w/v) TDI, $0.00001 \%$ (w/v) $\mathrm{CH}$, $0.00001 \%(\mathrm{w} / \mathrm{v})$ PI, $0.01 \%(\mathrm{w} / \mathrm{v}) \mathrm{DNCB}$ or $0.001 \%(\mathrm{w} / \mathrm{v})$ OXA were intratracheally instilled into both sensitized and non-sensitized mice (Table 1). Briefly, mice were anesthetized with a subcutaneously injection of midazolam $(4 \mathrm{mg} / \mathrm{kg})$, medetomidine $(0.1 \mathrm{mg} / \mathrm{kg})$ and butorphanol $(5 \mathrm{mg} / \mathrm{kg})$ in saline into the scruff of the neck. The trachea was then exposed surgically on the ventral side of the neck and intrapulumonary aerosolizer (PennCentury, PA, USA) was instilled via the mouth with monitoring of the exposed trachea for accurate instillation of chemicals. There were 8-10 mice per experimental group. The doses of dermal application were determined based on the previous studies (Dearman et al., 1998; Plitnick et al., 2003, 2005; Farraj et al., 2007) and our unpublished data, which showed that a topical application of chemicals had little effect on mice skin. In brief, chemicals at various concentrations were applied on mice skin, and then the skin was observed $24 \mathrm{hr}$ after application. The doses of intratracheal instillation were determined based on our pilot studies that chemicals at various concentrations were applied into lungs, and then the symptoms of mice were observed 24 
Cytokine profiles in BALF of mice exposed to chemical sensitizers

Table 1. Doses for dermal application and intratracheal instillation of chemicals

\begin{tabular}{|c|c|c|c|c|}
\hline \multirow[t]{2}{*}{ Chemical } & \multicolumn{2}{|c|}{$\begin{array}{l}\text { Dermal application } \\
\text { (Sensitization) }\end{array}$} & \multicolumn{2}{|c|}{$\begin{array}{c}\text { Intratracheal instillation } \\
\text { (Challenge) }\end{array}$} \\
\hline & Concentration $(\%)$ & Solvent & Concentration $(\%)$ & Solvent \\
\hline PA & 25 & $\mathrm{AOO}$ & 0.05 & DMSO/Olive oil \\
\hline MDI & 2 & $\mathrm{AOO}$ & 0.0001 & Olive oil \\
\hline TDI & 1 & $\mathrm{AOO}$ & 0.001 & Olive oil \\
\hline $\mathrm{CH}$ & 10 & DMSO & 0.00001 & DMSO/Olive oil \\
\hline PI & 5 & DMSO & 0.00001 & DMSO/Olive oil \\
\hline DNCB & 1 & $\mathrm{AOO}$ & 0.01 & Olive oil \\
\hline OXA & 1 & $\mathrm{AOO}$ & 0.001 & DMSO/Olive oil \\
\hline
\end{tabular}

For dermal application, PA, MDI, TDI, DNCB and OXA were dissolved in 4:1 acetone:olive oil (AOO) and CH and PI were dissolved in DMSO. For intratracheal instillation, PA, CH, PI and OXA were dissolved in DMSO and mixed with olive oil and MDI, TDI and DNCB were mixed with olive oil. $\mathrm{n}=8-10$ mice/group.

hr after application. The doses with little effect on mice symptoms were selected for intratracheal instillation of chemicals.

\section{Serum total IgE and BALF cytokines}

Mice were anesthetized with inhalation of diethyl ether $24 \mathrm{hr}$ after intratracheal instillation. Blood samples were collected from the abdominal aorta, and sera were stored frozen at $-20^{\circ} \mathrm{C}$ until measurement of total $\mathrm{IgE}$ levels. Mice were sacrificed after the collection of blood samples and the trachea was exposed surgically on the ventral side of the neck. An incision was made on the upper trachea and a needle was inserted to allow BALF to be obtained using $1.0 \mathrm{ml}$ of PBS ( $\mathrm{pH} 7.3$ ) containing $0.05 \mathrm{mM}$ ethylenediaminetetraacetic acid (Dojindo Laboratories, Kumamoto, Japan) and $1 \mathrm{mg} / \mathrm{ml}$ bovine serum albumin (Wako Pure Chemical Industry, Osaka, Japan). BALF samples were centrifuged $\left(800 \times \mathrm{g}, 10 \mathrm{~min}, 4^{\circ} \mathrm{C}\right)$ and the supernatants were frozen at $-80^{\circ} \mathrm{C}$ until cytokine measurement. Serum total IgE and BALF cytokines (G-CSF, IL-4, IL-5, IL-6, IL-12(p70), IL-13, IFN- $\gamma$, MCP-1 and TNF- $\alpha$ ) were measured by ELISA (Yamasa, Tokyo, Japan) and Bio-Plex (Bio-Rad, Tokyo, Japan), respectively, according to manufacturer's instructions. One-sided Student's $\mathrm{T}$ test was performed to assess whether the measurement values of sensitized groups were significantly greater than those of each control.

\section{RESULTS}

\section{Serum total $\lg \mathrm{E}$}

Mice were treated with chemicals as shown in Fig. 1. Non-sensitized mice were treated dermally with solvents and challenged intratracheally with the respective test chemicals as solvent controls. Remarkable differences in symptoms were not observed between sensitized and non-sensitized mice $24 \mathrm{hr}$ after intratracheal challenge. Sera were collected from dermally treated mice $24 \mathrm{hr}$ after intratracheal challenge. Respiratory sensitizers, PA, MDI, TDI, CH and PI, showed statistically significant increases in total serum IgE compared with each control (Fig. 2). As with these respiratory sensitizers, contact sensitizers DNCB and OXA caused increases in serum total IgE (Fig. 2).

\section{BALF cytokines}

BALF was obtained $24 \mathrm{hr}$ after intratracheal challenge (Fig. 1). Nine cytokines (G-CSF, IL-4, IL-5, IL-6, IL-12(p70), IL-13, IFN- $\gamma$, MCP-1 and TNF- $\alpha$ ) in BALF were measured by multiplex bead assays. Production of cytokines differed among chemicals, as shown in Fig. 3. The reproducibility of the results was confirmed by additional studies for each chemical (data not shown).

The level of IL-4 showed a statistically significant increase in PA-sensitized mice compared with the control. However, the other chemicals did not cause any increase of IL-4 in sensitized mice. Intratracheal challenge with $\mathrm{CH}$ also resulted in elevated IL-4 in BALF, but was observed in both CH-sensitized and control mice. Thus, it was considered that the responses were not based on immune reactions. $\mathrm{PA}$ and $\mathrm{CH}$, but not the other chemicals used in this study, caused statistically significant increases in the levels of IL-5 compared with controls. PA, TDI, CH, PI and DNCB induced statistically significant increases in the levels of IL-6. Elevation of IL-12(p70) levels was statistically significant with PA, MDI and TDI. Similarly 


\section{T. Mori et al.}

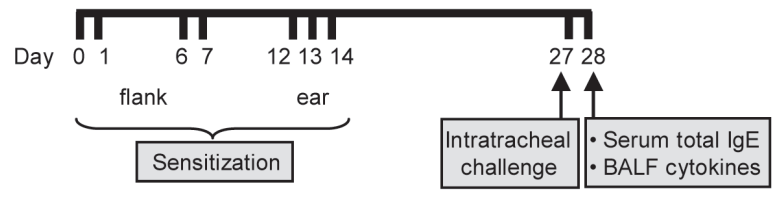

Fig. 1. Experimental design. Fifty $\mu 1$ aliquots of chemicals were applied on each shaved flank on days $0,1,6$ and 7. On days 12,13 and $14,25 \mu 1$ of the same chemical was applied on the dorsum of both ears. On day 27, $50 \mu \mathrm{l}$ of the chemical was intratracheally instilled and serum collection and bronchoalveolar lavage were performed $24 \mathrm{hr}$ thereafter. $\mathrm{n}=8-10$ mice/group.

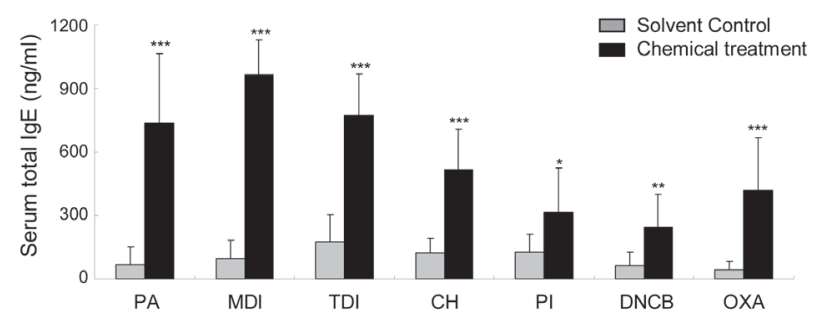

Fig. 2. Serum total IgE, measured by ELISA. ${ }^{*} \mathrm{P}<0.05,{ }^{*} \mathrm{p}$ $<0.01, * * * \mathrm{p}<0.001$ compared with each respective control group. $\mathrm{n}=8-10$ mice/group.
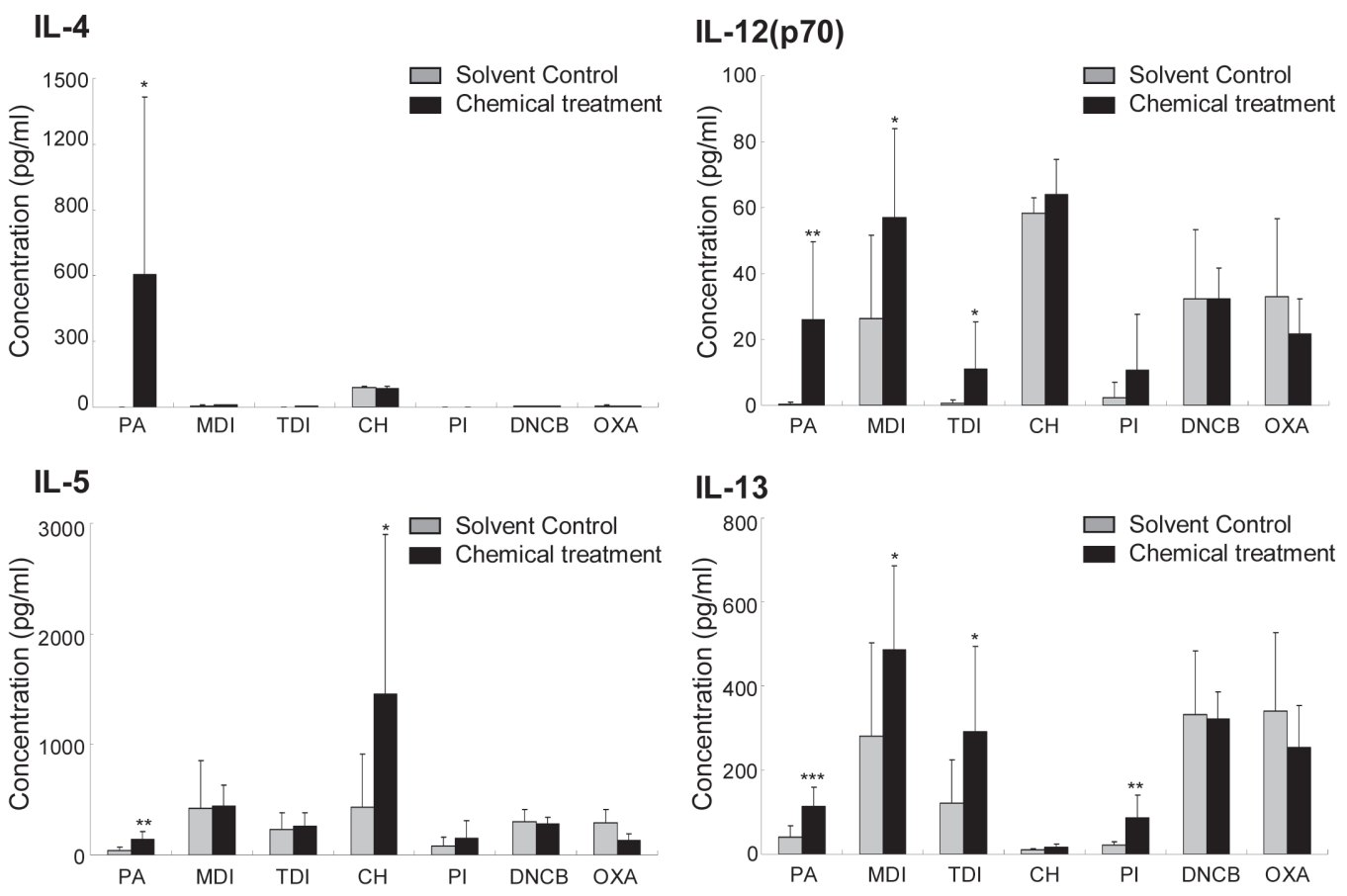

\section{IL-6}

IFN- $\gamma$
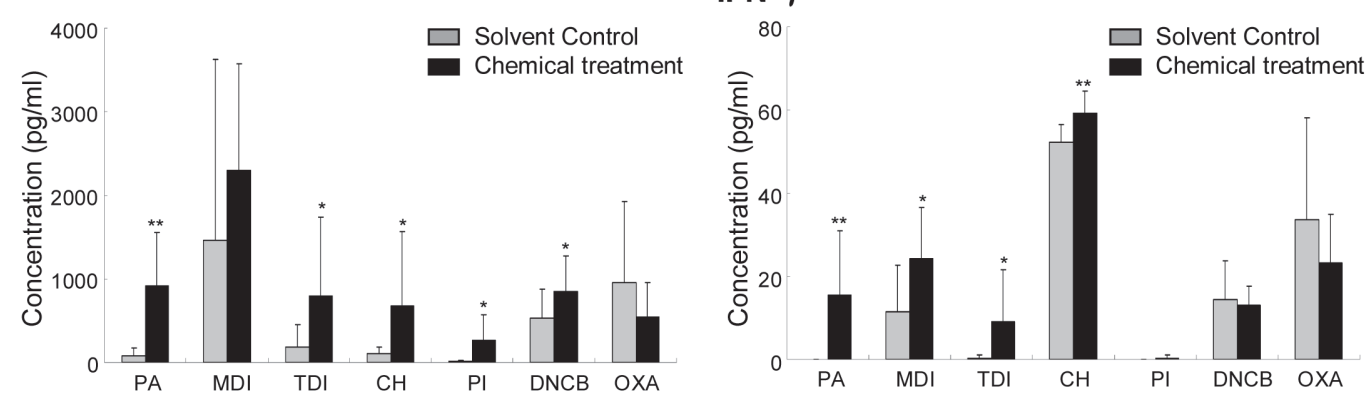

Fig. 3. BALF cytokines. IL-4, IL-5, IL-6, IL-12(p70), IL-13, IFN- $\gamma$, MCP-1, G-CSF and TNF- $\alpha$ were measured using a multiple cytokine assay system (Bio-Plex). ${ }^{*} \mathrm{P}<0.05,{ }^{* *} \mathrm{p}<0.01,{ }^{* * *} \mathrm{p}<0.001$ compared with each respective control group. $\mathrm{n}=8-10$ mice/group. 
Cytokine profiles in BALF of mice exposed to chemical sensitizers

IL-13 was found to be increased with PA, MDI, TDI and PI, and IFN- $\gamma$ with PA, MDI, TDI and $\mathrm{CH}$. Only PA and TDI caused statistically significant increase in MCP-1. All respiratory sensitizers tested in this study (PA, MDI, TDI, CH and PI) induced statistically significant increases in the levels of G-CSF compared with controls. In contrast, no such change was evident with contact sensitizers (DNCB and OXA). PA, MDI and TDI caused statistically significant increase in TNF- $\alpha$.

\section{DISCUSSION}

In this study, five respiratory sensitizers (PA, MDI, TDI, $\mathrm{CH}$ and PI) induced statistically significant increases in total serum IgE (Fig. 2), but so did both contact sensitizers, DNCB and OXA, similarly tested (Fig. 2). Previous reports also showed that dermal application of not only respiratory sensitizers but additionally a contact sensitizer DNCB increased serum total IgE in mice (Ban and Hettich, 2001; Selgrade et al., 2006). While serum IgE is considered to be one of the triggers of chemical-induced asthma (Holsapple et al., 2006; Arts and Kuper, 2007), epidemiological studies have suggested that IgE inde- pendent responses may also be involved (Kimber et al., 1998). The available data thus suggest that increase in total serum IgE is related to immune reactions, but itself can not be used as an indicator to discriminate between respiratory and contact sensitizers, at least in dermally sensitized mice.

Cytokine profiling of dermally sensitized mice consistently demonstrated their effectiveness as indicators of respiratory sensitizers (Dearman et al., 2000; Dearman and Kimber, 2001). Dermal application of PA, MDI, TDI, CH and PI caused Th2 immune responses in mice (Dearman et al., 1998; Plitnick et al., 2003, 2005; Farraj et al., 2007). We therefore used these chemicals to analyze immune responses in lungs. The present study showed that all five induced statistically significant increases in BALF cytokines as a result of dermal sensitization and intratracheal challenge (Fig. 3). However, differences in BALF cytokine profiles were observed, PA, MDI, TDI and PI for example causing statistically significant increases in IL-13, whereas no change was apparent with $\mathrm{CH}$. Earlier work indicated skin exposures of respiratory sensitizers such as PA, MDI, TDI, $\mathrm{CH}$ and PI to induce Th2 responses featuring increased levels of IL-4, IL-5 and
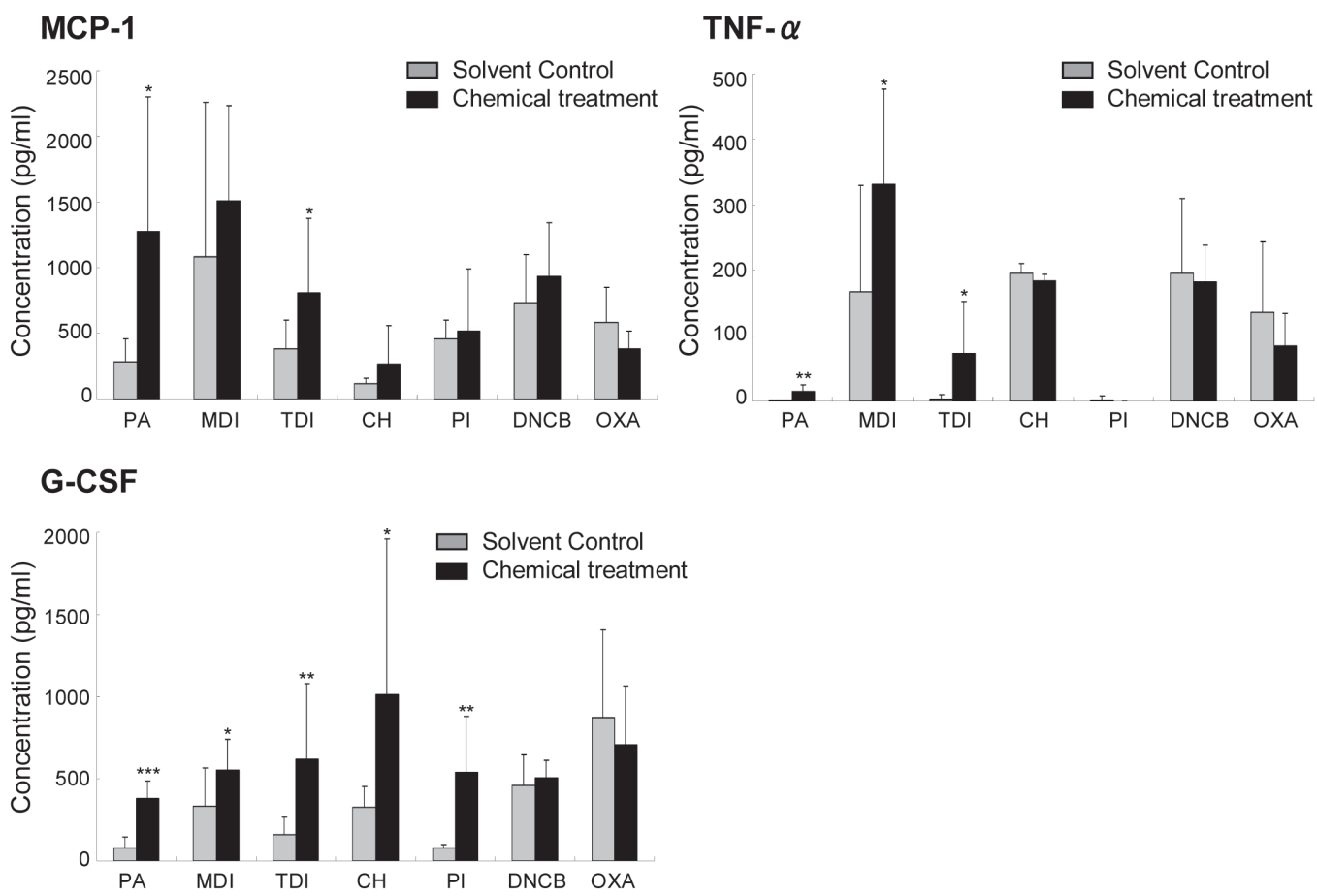

Fig. 3. (Continued.) 
Table 2. Summary of results

\begin{tabular}{lccccccc}
\hline Chemical & PA & MDI & TDI & CH & PI & DNCB & OXA \\
\hline Serum total IgE & +++ & +++ & +++ & +++ & + & ++ & +++ \\
\hline BALF cytokine & & & & & & & \\
IL-4 & + & - & - & - & - & - & - \\
IL-5 & ++ & - & - & + & - & - & - \\
IL-6 & ++ & - & + & + & + & + & - \\
IL-12 (p70) & ++ & + & + & - & - & - & - \\
IL-13 & +++ & + & + & - & ++ & - & - \\
IFN- $\gamma$ & ++ & + & + & ++ & - & - & - \\
MCP-1 & + & - & + & - & - & - & - \\
G-CSF & +++ & + & ++ & + & + & - & - \\
TNF- $\alpha$ & ++ & + & + & - & - & - \\
\hline
\end{tabular}

The measurement results of chemical-sensitized mice were compared with those of each control. + indicates a statistically significant increase ( $+\mathrm{P}<0.05,++\mathrm{P}<0.01$ and $+++\mathrm{P}<0.001)$; - indicates no change. $\mathrm{n}=8-10$ mice/group.

IL-13 in lymph nodes. The elevation of IL-4 levels was due to increases in IL-4 producing T cells in lymph nodes (Dearman et al., 2000; Dearman and Kimber, 2001). However, our results showed that IL-5 and IL-13 were increased in BALF by two and four examples, respectively, whereas IL-4 showed a statistically significant increase only in BALF of PA sensitized mice (Fig. 3). These results imply that IL-4 producing $\mathrm{T}$ cells were increased in lymph nodes but not in lungs of mice sensitized with MDI, TDI, CH or PI. In addition to Th2 cytokines, production of Th1 cytokines like IL-12(p70) and IFN- $\gamma$ was elevated by several of our respiratory sensitizers (Fig. 3). It seems that the doses and frequencies of dermal application influence the Th1/Th2 balance and induce complex immune responses with Th1 and Th2 cytokines. Our results indicate that we may need to analyze a panel of several cytokines to assess immune responses.

Respiratory sensitizers increased Th2 cytokines in BALF (Fig. 3), which showed that immune responses were caused in lungs. The present study showed that all five respiratory sensitizers induced statistically significant increase in G-CSF, but not DNCB and OXA (Fig. 3). G-CSF functions as a stimulatory factor for neutrophils, which are reported to be involved in chemical-induced asthma in man (Park et al., 1999). Increase in neutrophils was also observed in animal models with dermal sensitization (Matheson et al., 2005; Vanoirbeek et al., 2004). Influx of eosinophils observed in human and animal models was also appears related to allergic asthma. However, our study showed that IL-5, which is closely related to the influx of eosinophils in lungs, was only increased by
PA and CH (Fig. 3). The animal model used in this study featured intratracheal challenge only once and BALF was collected $24 \mathrm{hr}$ after the challenge, to detect immune responses at an early stage. A more sustained challenge may be required to generally induce influx of eosinophils. Thus, it was considered that neutrophils, rather than eosinophils, may be effective indicators in this topically intratracheal challenge model. Notably, G-CSF was increased by all respiratory sensitizers used in this study (Table. 2). The findings thus suggest that G-CSF and related cytokines could be effective to identify respiratory sensitizers in animal models.

\section{REFERENCES}

Arts, J.H. and Kuper, C.F. (2007): Animal models to test respiratory allergy of low molecular weight chemicals: a guidance. Methods, 41, 61-71.

Ban, M. and Hettich, D. (2001): Relationship between IgE positive cell numbers and serum total IgE levels in mice treated with trimellitic anhydride and dinitrochlorobenzene. Toxicol. Lett., 118, 129-137.

Ban, M., Morel, G., Langonné, I., Huguet, N., Pépin, E. and Binet, S. (2006): TDI can induce respiratory allergy with Th2-dominated response in mice. Toxicology, 218, 39-47.

Boverhof, D.R., Billington, R., Gollapudi, B.B., Hotchkiss, J.A., Krieger, S.M., Poole, A., Wiescinski, C.M. and Woolhiser, M.R., (2008): Respiratory sensitization and allergy: Current research approaches and needs. Toxicol. Appl. Pharm., 226, 1-13.

Dearman, R.J., Basketter, D.A. and Kimber, I. (1998): Selective induction of Type 2 cytokines following topical exposure of mice to platinum salts. Food and Chemical Toxicology, 36, 199207.

Dearman, R.J., Warbrick, E.V., Humphreys, I.R. and Kimber, I. 
Cytokine profiles in BALF of mice exposed to chemical sensitizers

(2000): Characterization in mice of the immunological properties of five allergenic acid anhydrides. J. Appl. Toxicol., 20, 221230.

Dearman, R.J. and Kimber, I. (2001): Cytokine fingerprinting and hazard assessment of chemical respiratory allergy. J. Appl. Toxicol., 21, 153-163.

Farraj, A.K., Boykin, E., Haykal-Coates, N., Gavett, S.H., Doerfler, D. and Selgrade, M. (2007): Th2 cytokines in skin draining lymph nodes and serum IgE do not predict airway hypersensitivity to intranasal isocyanate exposure in mice. Toxicol. Sci., 100, 99-108.

Fukuyama, T., Ueda, H., Hayashi, K., Tajima, Y., Shuto, Y., Saito, T.R., Harada, T. and Kosaka, T. (2008): Use of long term dermal sensitization followed by intratracheal challenge method to identify low-dose chemical-induced respiratory allergic responses in mice. Toxicol. Lett., 181, 163-170.

Holsapple, M.P., Jones, D., Kawabata, T.T., Kimber, I., Sarlo, K., Selgrade, M.K., Shah, J. and Woolhiser, M.R. (2006): Assessing the potential to induce respiratory hypersensitivity. Toxicol. Sci., 91, 4-13.

Hur, G.Y., Sheen, S.S., Kang, Y.M., Koh, D.H., Park, H.J., Ye, Y.M., Yim, H.E., Kim, K.S. and Park, H.S. (2008): Histamine release and inflammatory cell infiltration in airway mucosa in methylene diphenyl diisocyanate (MDI)-induced occupational asthma. J. Clin. Immunol., 28, 571-580.

Kimber, I., Warbrick, E.V. and Dearman, R.J. (1998): Chemical respiratory allergy, IgE and the relevance of predictive test methods: a commentary. Hum. Exp. Toxicol., 17, 537-540.

Kimber, I., Agius, R., Basketter, D.A., Corsini, E., Cullinan, P., Derman, R.J., Gimenez-Arnau, E., Greenwell, L., Hartung, T., Kuper, F., Maestrelli, P., Roggen, E. and Rovida, C. (2007): Chemical respiratory allergy: opportunities for hazard identification and characterisation. The report and recommendations of ECVAM workshop 60. Altern. Lab. Anim., 35, 243-265.

Leroyer, C., Perfetti, L., Cartier, A. and Malo, J.L. (1998): Can reactive airways dysfunction syndrome (RADS) transform into occupational asthma due to "sensitisation" to isocyanates? Thorax., 53, 152-153.
Mapp, C.E., Boschetto, P., Maestrelli, P. and Fabbri, L.M. (2005): Occupational asthma. Am. J. Respir. Crit. Care. Med., 172, 280305.

Matheson, J.M., Johnson, V.J., Vallyathan, V. and Luster, M.I. (2005): Exposure and immunological determinants in a murine model for toluene diisocyanate (TDI) asthma. Toxicol. Sci., 84, 88-98.

Park, H., Jung, K., Kim, H., Nahm, D. and Kang, K. (1999): Neutrophil activation following TDI bronchial challenges to the airway secretion from subjects with TDI-induced asthma. Clin. Exp. Allergy., 29, 1395-1401.

Plitnick, L.M., Loveless, S.E., Ladics, G.S., Holsapple, M.P., Smialowicz, R.J., Woolhiser, M.R., Anderson, P.K., Smith, C. and Selgrade, M.J. (2003): Identifying airway sensitizers: cytokine mRNA profiles induced by various anhydrides. Toxicology, 193, 191-201.

Plitnick, L.M., Loveless, S.E., Ladics, G.S., Holsapple, M.P., Smialowicz, R.J., Woolhiser, M.R., Anderson, P.K., Smith, C. and Selgrade, M.J. (2005): Cytokine mRNA profiles for isocyanates with known and unknown potential to induce respiratory sensitization. Toxicology, 207, 487-499.

Selgrade, M., Boykin, E.H., Haykal-Coates, N., Woolhiser, M.R., Wiescinski, C., Andrews, D.L., Farraj, A.K., Doerfler, D.L. and Gavett, S.H. (2006): Inconsistencies between cytokine profiles, antibody responses, and respiratory hyperresponsiveness following dermal exposure to isocyanates. Toxicol. Sci., 94, 108-117.

Tarlo, S.M. (2003): Occupational asthma: a valid model for adult asthma? Curr. Opin. Allergy. Clin. Immunol., 3, 91-94.

Van, Och. F.M., Van, Loveren. H., De, Jong. W.H. and Vandebriel, R.J. (2002): Cytokine production induced by lowmolecular-weight chemicals as a function of the stimulation index in a modified local lymph node assay: an approach to discriminate contact sensitizers from respiratory sensitizers. Toxicol. Appl. Pharmacol., 184, 46-56.

Vanoirbeek, J.A., Tarkowski, M., Ceuppens, J.L., Verbeken, E.K., Nemery, B. and Hoet, P.H. (2004): Respiratory response to toluene diisocyanate depends on prior frequency and concentration of dermal sensitization in mice. Toxicol. Sci., 80, 310-321. 